\title{
An Action-Based Approach to Improving Pain Management in Long-Term Care*
}

\author{
Sharon Kaasalainen, 1,2 Kevin Brazil, 3,4 Esther Coker, ${ }^{1,5}$ Jenny Ploeg, ${ }^{1}$ Ruth Martin-Misener, ${ }^{6}$ \\ Faith Donald, 7 Alba DiCenso, 1,3 Thomas Hadjistavropoulos, ${ }^{8}$ Lisa Dolovich, 2,3,4,9 \\ Alexandra Papaioannou, ${ }^{9}$ Anna Emili, ${ }^{2}$ and Tim Burns ${ }^{10}$
}

\begin{abstract}
RÉSUMÉ
Objectif : Les objectifs de cette étude étaient double : (1) d'explorer les obstacles à la gestion de la douleur et ceux associés à la mise en œuvre d'un programme de gestion de la douleur en soins de longue durée (SLD) et (2) de développer une approche interprofessionelle afin d'améliorer la gestion de la douleur en soins de longue durée.

Méthodes : Une étude de cas a inclus tous les deux éléments, quantitatifs et qualitatifs. Nous avons recueilli des données sur deux sites de SLD en utilisant six groupes de discussion pour les infirmières autorisées et fournisseurs de soins nonréglementés, et 10 entretiens avec des autres groupes de fournisseurs de soins de santé, de l'administration, et des résidents. Nous avons examiné les documents et administré un sondage à court aux participants à l'étude pour évaluer les perceptions des obstacles à la gestion de la douleur.

Résultats : Les résultats ont révélé des obstacles à la gestion efficace de la douleur au niveau des soins de longue durée des résidents et des familles, des fournisseurs de soins de santé, et des systèmes de santé. Nous avons ensuite élaboré un modèle à six niveaux, avec les interventions proposées pour surmonter ces obstacles.

Conclusions : Ce modèle peut guider le développement d'approches novatrices pour améliorer la gestion de la douleur dans les établissements de soins de longue durée. [To spell out seems preferable to using the acronym here-Trans.]
\end{abstract}

\section{ABSTRACT}

Purpose: The study purposes were twofold: (1) to explore barriers to pain management and those associated with implementing a pain management program in long-term care (LTC); and (2) to develop an interprofessional approach to improve pain management in LTC.

Methods: A case study approach included both qualitative and quantitative components. We collected data at two LTC sites using six focus groups for the licensed nurses and unregulated care providers, and 10 interviews with other health care provider groups, administration, and residents. We reviewed documents and administered a short survey to study participants to assess perceptions of barriers to pain management.

Results: The findings revealed barriers to effective LTC pain management at the resident/family, health care provider, and system levels. We then developed a six-tiered model with proposed interventions to address these barriers.

Conclusions: This model can guide the development of innovative approaches to improving pain management in LTC settings.

1 School of Nursing, McMaster University

2 Department of Family Medicine, McMaster University

3 Department of Clinical Epidemiology and Biostatistics, McMaster University

4 Centre for Evaluation of Medicine, St Joseph's Healthcare

5 Hamilton Health Sciences

6 School of Nursing, Dalhousie University

7 Daphne Cockwell School of Nursing, Ryerson University

8 Psychology Department, University of Regina

9 Department of Medicine, McMaster University

10 Ontario Ministry of Health and Long-Term Care

* This study was supported by a grant from the Canadian Institutes of Health Research and the Ontario Ministry of Health and Long Term Care. The authors are grateful for the willingness of the study participants to share their time and expertise with us for this study. This project benefited greatly from the commitment and expertise of research assistants Nancy Carter and Kenzie Greenhalgh. The first author (SK) was supported by a Canadian Health Services Research Foundation (CHSRF) Postdoctoral Fellowship and an Ontario Ministry of Health and Long-Term Care Career Scientist award, at separate times, during this study and the writing of this article. Dr. Alba DiCenso holds a Canadian Health Services Research Foundation (CHSRF)/Canadian 
Institutes for Health Research/CIHR Chair in Advanced Practice Nursing. Dr. Thomas Hadjistavropoulos' work is supported, in part, by the RBC Foundation. Faith Donald was supported by a CHSRF Postdoctoral Fellowship.

Manuscript received: / manuscrit reçu : 04/05/09

Manuscript accepted: / manuscrit accepté : 31/05/10

Mots clés :

Keywords: pain management, long-term care, interdisciplinary, barriers assessment

Correspondence and requests for offprints should be sent to / La correspondance et les demandes de tirés-à-part doivent être adressées à:

Sharon Kaasalainen, R.N., Ph.D.

Faculty of Health Sciences, 3N25F

McMaster University 1200 Main Street West

Hamilton, ON

L8N $3 Z 5$

(kaasal@mcmaster.ca)

As many as 83 per cent of older adults living in longterm care (LTC) experience pain (Desbiens, MuellerRizner, Connors, Hamel, \& Wenger, 1997; Ferrell, Ferrell, \& Osterweil, 1990; Fox, Raina, \& Jadad, 1999; Moulin, Clark, Speechley, \& Morley-Forster, 2002; Proctor \& Hirdes, 2001; Simons \& Malabar, 1995). Unresolved pain can lead to decreased functional abilities, depression, loneliness, impaired mobility, sleep disturbances, anxiety, and dissatisfaction with life (Ferrell et al., 1990; Ross \& Crook, 1998). Pain in LTC residents is often under-assessed and under-treated (Horgas \& Tsai, 1998; Kaasalainen, Middleton, Knezacek, Stewart, Hartley, Ife et al., 1998). Moreover, pain management decisions of health care providers appear to be influenced by a number of challenges that exist within LTC, including lack of accessible services, shortcomings of self-report methods of pain assessment, communication barriers between patients and health professionals, reluctance of nurses and physicians to use opioids, and lack of knowledge about pain management, particularly for residents who have dementia (Kaasalainen, Coker, Dolovich, Papaioannou, Hadjistavropoulos, Emili et al., 2007; Martin, Williams, Hadjistavropoulos, Hadjistavropoulos, \& MacClean, 2005).

In response to the growing concern about inadequate pain management in LTC, leading experts in pain management and public policy in both Canada and the United States have made a number of recommendations to improve pain management in LTC (Hadjistavropoulos, Marchildon, Fine, Herr, Palley, Kaasalainen et al., 2009). Also, a number of pain management clinical practice guidelines (CPGs) have been developed by professional organizations, including the Registered Nurses Association of Ontario (RNAO, 2002a), American Geriatrics Society (AGS, 1998, no date), and the American Medical Directors Association (AMDA, 2003), to assist staff in their decision making involving pain management.
We developed a pain protocol, on the basis of CPGs, which includes a series of processes and supporting tools that guide recognition, assessment, treatment, and monitoring of pain (AMDA, 2003). These more specific steps are largely consistent with a general clinical model that has been suggested in the literature (Hadjistavropoulos et al., 2009). Initially, residents are screened for the presence of pain using simple questions like "Are you having pain now?"; for those who cannot verbally communicate their pain, unlicensed care providers (UCPs) are asked to screen using a list of pain indicators (AMDA, 2003), while licensed nurses use recommended evidence-based screening tools (Fuchs-Lacelle \& Hadjistavropoulos, 2004; Kaasalainen \& Crook, 2003). If residents are in pain, a more thorough assessment follows using the family as proxy if needed. The protocol prompts further activities, such as a physical assessment to help identify the cause of pain, and provides indicators for when specialists should be consulted.

Depending on the severity and cause of pain, pain treatment algorithms (Medical College of Wisconsin, 2000) can be used to guide decision making regarding appropriate non-pharmacological and pharmacological approaches. A Nurse Worksheet for Phone Call Interventions (Medical College of Wisconsin, 2000) is used to guide the collection of pertinent information about the resident's pain for communication to the physician. A pain flow sheet is used to facilitate interdisciplinary communication and support documentation of pain and its treatments.

The implementation of such a protocol in LTC can be challenging, as there are large numbers of UCPs, multiple physicians with little on-site time due to competing office and hospital demands, intermittent clinical pharmacist visits, and few (if any) advanced practice nurses on staff (Bakerjian, 2008). Innovative ways of facilitating 
the implementation of CPGs are needed to enhance knowledge uptake and changes in clinical practice, particularly regarding pain management.

In light of the complexity and challenges of implementing CPGs, a number of toolkits have been developed to facilitate a systematic and well-planned implementation process (AMDA, 2004; RNAO, 2002b). Both the RNAO and AMDA toolkits outline a stepwise approach to CPG implementation, including an environmental scan to identify barriers and develop strategies to overcome them in the implementation process. It is important to facilitate stakeholder input and "buy$i^{\prime \prime}$ at the outset by including stakeholders in the decision making before such a protocol is implemented (RNAO, 2002b). In addition, the AMDA recommends that pertinent policies and procedures need to be reviewed and revised to help standardize the desired approach to pain management (AMDA, 2004).

Efforts are needed to improve pain management in LTC using creative strategies aimed at addressing common and unique barriers that exist within current practice. Innovations within LTC need to consider these existing barriers, as well as those imposed by the demands of the intervention itself. To do this, a comprehensive approach must be developed that addresses these barriers within an interprofessional, multi-level context.

The purpose of this study was twofold: (1) to identify barriers to pain management in general, along with barriers to implementing a pain protocol in LTC; and (2) to develop an interprofessional model reflecting an approach to improve pain management in LTC in light of those barriers. This study was the first phase of a larger study in which a pain protocol was implemented and evaluated in LTC. It was anticipated that having knowledge of barriers beforehand, and engaging staff in customizing processes to fit with some of their current practices, would increase staff buy-in. Based on findings, we proposed an action-based, system-wide, interdisciplinary model to improve pain management. The findings of the barriers assessment, along with this model, are reported here.

\section{Methods}

We used a case study approach that incorporated both qualitative and quantitative methodologies to explore the barriers to the implementation of a pain protocol (Yin, 1984). The case study approach is particularly helpful when the study question deals with the "how" and "why" of an intervention (Crabtree \& Miller, 1999).

\section{Sampling}

Purposive sampling was used to collect data at two LTC homes in the Hamilton, Ontario, area. Six focus groups were held in total: one at each site with registered nurses (RNs), one with registered practical nurses (RPNs; only one site employed RPNs), one at each site with UCPs, and one that included physicians from both facilities.

Brown (1999) suggested using a homogenous sample for focus groups to ensure a level of compatibility among participants and reduce the risk of power imbalances and lack of respect for differing opinions. Therefore, physicians, RNs, RPNs, and UCPs were interviewed in uni-disciplinary groups as they may have had different perspectives or they may have had issues related to the inter-group working relationships that affected their perceptions about current pain management practices.

Because pharmacists and physiotherapists were few in number at each site, we conducted individual interviews with them rather than with focus groups. We interviewed two pharmacists and two physiotherapists. We also interviewed two administrators, two directors of care, and two residents. Members of administration (one administrator and one director of care from each site) were chosen as key informants to help acquire a sense of the organizational culture at each facility (Gilchrist \& Williams, 1999). Two residents were identified by the advanced practice nurses as key informants who were able to communicate "the way it is" in LTC and were most knowledgeable about the resident culture (Gilchrist \& Williams, 1999).

\section{Data Collection}

The advanced practice nurse at each home recruited individuals to participate in the focus groups that were held on-site for the participants' convenience. A trained moderator facilitated focus group discussions. A second person observed the non-verbal communication in the group and took field notes during the session (Morgan, 1997). Focus groups began with welcoming participants, outlining the purpose of the discussion, setting the parameters of discussion (e.g., length, audio taping, transcribing), and assuring confidentiality of their responses outside the group (Morgan, 1997).

For the individual and key informant interviews, a research assistant first asked if the individual or informant would be willing to participate in a one-on-one interview. If agreeable, the participants met for respective 30-minute face-to-face interviews at a convenient location.

At the beginning of each interview (i.e., focus group, individual, or key informant), participants were asked to complete a 15-item questionnaire that assessed the extent to which they felt each potential barrier to pain management (e.g., residents' reluctance to report pain 
or inability to report pain) existed in their facility (Jones, Fink, Pepper, Hutt, Vojir, Scott et al., 2004). Each barrier was scored on a scale ranging from 1 (not a problem) to 4 (major problem). This questionnaire had been developed and piloted in a study in the United States that surveyed over 300 licensed nursing staff across 12 nursing homes (Jones et al., 2004).

Next, the moderator used an interview guide (available upon request) to generate discussion about how staff currently manage pain in their practice and barriers they face in managing pain in LTC residents. Finally, the moderator provided copies of the pain protocol, explained the protocol steps, and asked for feedback about it (i.e., attitudes, concerns, and intention to use the protocol in their practice) while brainstorming with them possible ways of integrating it into their current practice. Finally, we reviewed the existing pain management policies and procedures at the two LTC sites and critiqued them according to the guidelines outlined in the AMDA implementation toolkit, with a particular focus on who should be responsible for completing each component (AMDA, 2004).

\section{Data Analysis}

\section{Quantitative}

The survey data were analyzed using descriptive statistics. The mean scores and standard deviations were calculated for each item using the total sample. Separate mean scores were also calculated for each type of health care provider or member of administration for descriptive purposes only.

\section{Qualitative}

Data from the focus groups and interviews were transcribed and analyzed using the software program $\mathrm{N}$ Vivo 7.0 (QSR International Ltd., 2007). It assisted with the sorting and analysis of audio files, and working with transcripts to facilitate the coding and organizing of themes within the data. Concepts that emerged from the data were labeled, categorized, and coded (Patton, 2000; Sandelowski, 2000). To examine focus group data, we used a descriptive analysis technique. This technique places particular emphasis on examining (a) statements that seem to evoke conflict; (b) contradictions in the discussion; (c) common experiences expressed; (d) alliances formed among group members during discussion; and (e) topics that produced consensus (Duggleby, 2005; Stevens, 1996).

Initial coding of each transcript was done independently by two investigators to foster credibility and dependability. Discrepancies were reviewed by the investigators and discussed until consensus was reached. As the data were analyzed, changes were made to the interview guide to reflect emerging themes.
We used a number of methods to triangulate the data to improve the credibility of the findings. First, data triangulation was enhanced using multiple data sources and strategies (i.e., collecting data from focus groups, individual interviews, and documents at two different LTC facilities and from professionals from different disciplines). Second, member checking or "recycling interpretation" was performed such that after their interviews, each informant was asked to review a twopage summary of key findings and provide comments relating to the investigators' interpretation of the interview data (Crabtree \& Miller, 1999). Investigator triangulation was used to minimize any idiosyncratic biases. Each transcript was independently analyzed by two investigators. Our research team used an iterative process, meeting regularly to review progress and ensure that in-depth data interpretation was occurring.

\section{Results}

Characteristics of the Setting and Sample

Both LTC homes participating in this study were medium-sized facilities (e.g., one home with 125 beds, the other with 219 beds) located in urban settings. Both homes provided nursing and personal care on a 24hour basis. One was funded on a for-profit basis and the other one was not-for-profit.

In total, there were 53 participants: 45 in focus groups (15 RNs, 6 RPNs, 20 UCPs, four physicians) and 8 involved in one-on-one interviews (four members of administration, two physiotherapists, two pharmacists). The majority of health care providers $(71 \%)$ were female with the lowest percentages of females in the physician group $(33 \%)$ and pharmacist group $(0 \%)$. The average age of all participants was $49.3(S D=13.54)$. On average, health care providers and members of administration had been working in their current positions for 8 years $(S D=7.6)$ and in LTC for 11 years $(S D=9.93)$. Members of administration tended to be older $(M=52.3$ years; $S D=5.2)$ and had been working in LTC for a longer period of time $(M=18.38$ years; $S D=15.96)$ than the health care providers $(M=47$ years; $S D=12.7 ; M=10$ years; $S D=9.12)$.

Survey Findings of Barriers to Pain Management in LTC

Participants rated a number of barriers to effectively managing pain in LTC (see Table 1). The barrier rated as most problematic was residents' inability to report pain; it was ranked the highest by RNs, RPNs, UCPs, physicians, and members of administration. This was followed by residents' reluctance to report pain, and staff not having adequate time to assess for pain. Other prominent barriers included, in descending order: families' concerns about side effects, residents' reluctance to take analgesics, inadequate communication 
Table 1: Survey findings of barriers to pain management in long-term care (LTC)

\begin{tabular}{|c|c|c|c|c|c|c|c|c|c|c|c|c|c|c|c|c|}
\hline \multirow[t]{3}{*}{ Barrier } & \multicolumn{16}{|c|}{ Ratings of Barriers ${ }^{a}$} \\
\hline & \multicolumn{2}{|c|}{$N=53$} & \multicolumn{2}{|c|}{$n=15$} & \multicolumn{2}{|c|}{$n=6$} & \multicolumn{2}{|c|}{$n=20$} & \multicolumn{2}{|c|}{$n=2$} & \multicolumn{2}{|c|}{$n=2$} & \multicolumn{2}{|l|}{$n=4$} & \multicolumn{2}{|c|}{$n=4$} \\
\hline & $M$ & $S D$ & $M$ & $S D$ & $M$ & $S D$ & $M$ & $S D$ & $M$ & $S D$ & $M$ & $S D$ & $M$ & $S D$ & $M$ & $S D$ \\
\hline $\begin{array}{l}\text { 1. Residents' inability to } \\
\text { report pain }\end{array}$ & 3.04 & 0.94 & 3.2 & 0.86 & 2.8 & 0.96 & 3.0 & 1.08 & 2.5 & 0.71 & 2.5 & 0.71 & 4.0 & 0.00 & 3.3 & 0.96 \\
\hline $\begin{array}{l}\text { 2. Residents' reluctance to } \\
\text { report pain }\end{array}$ & 2.64 & 0.96 & 3.0 & 0.92 & 2.3 & 1.26 & 2.7 & 0.86 & 1.5 & 0.71 & 2.5 & 2.12 & 2.0 & 0.82 & 3.0 & 0.82 \\
\hline $\begin{array}{l}\text { 3. Inadequate time for staff } \\
\text { to assess pain }\end{array}$ & 2.62 & 1.16 & 2.8 & 1.12 & 1.5 & 1.00 & 2.5 & 1.24 & 2.5 & 2.12 & 3.5 & 0.71 & 3.5 & 0.50 & 2.8 & 0.50 \\
\hline $\begin{array}{l}\text { 4. Families' concerns about } \\
\text { side effects }\end{array}$ & 2.48 & 0.91 & 2.4 & 1.02 & 1.8 & 0.50 & 2.8 & 1.02 & 2.5 & 0.71 & 2.0 & 0.00 & 2.4 & 0.50 & 2.5 & 0.58 \\
\hline $\begin{array}{l}\text { 6. Inadequate communication } \\
\text { among nursing staff }\end{array}$ & 2.06 & 1.14 & 1.9 & 0.77 & 2.5 & 1.00 & 2.0 & 1.08 & 1.5 & 0.71 & 2.5 & 0.71 & 2.5 & 0.58 & 2.3 & 0.50 \\
\hline $\begin{array}{l}\text { 8. Inadequate staff knowledge } \\
\text { about pain management }\end{array}$ & 2.02 & 0.98 & 1.8 & 0.97 & 2.0 & 0.82 & 1.8 & 1.09 & 2.0 & 1.41 & 3.0 & 0.00 & 2.8 & 0.58 & 2.8 & 0.50 \\
\hline 9. Residents' fear of side effects & 1.92 & 0.83 & 1.9 & 0.66 & 1.8 & 0.50 & 2.0 & 1.15 & 2.5 & 0.71 & 2.0 & 0.00 & 1.9 & 0.50 & 2.0 & 0.00 \\
\hline $\begin{array}{l}\text { 10. Inadequate communication } \\
\text { between nurses and } \\
\text { physicians }\end{array}$ & 1.75 & 1.08 & 1.5 & 0.58 & 1.8 & 1.50 & 1.6 & 1.23 & 1.5 & 0.71 & 2.5 & 0.71 & 2.75 & 0.50 & 1.6 & 0.58 \\
\hline $\begin{array}{l}\text { 11. Nurses' reluctance to } \\
\text { administer analgesics }\end{array}$ & 1.66 & 0.82 & 1.4 & 0.50 & 2.0 & 1.41 & 1.6 & 0.94 & 1.5 & 0.71 & 2.0 & 0.00 & 2.0 & 0.82 & 2.3 & 0.50 \\
\hline $\begin{array}{l}\text { 12. Influence of federal } \\
\text { and/or state regulations }\end{array}$ & 1.59 & 1.07 & 1.4 & 0.85 & 1.5 & 1.0 & 1.8 & 1.45 & 1.5 & 0.71 & 2.0 & 0.00 & 1.0 & 0.50 & 1.8 & 1.75 \\
\hline
\end{tabular}

a Possible range of scores: 1 = no problem; 2 = minor problem; $3=$ moderate problem; $4=$ major problem

Admin $=$ Members of Administration

MD = Physicians

Pharm $=$ Pharmacists

PT $=$ Physiotherapists

RN = Registered Nurses

RPN = Registered Practical Nurses

among nursing staff, nurses' concerns about side effects of pain medications, and inadequate staff knowledge about pain management.

\section{Overview of Qualitative Findings}

During the interviews (i.e., focus groups, individuals, and key informants), participants described a number of barriers existing in their workplace that precluded optimal pain management for LTC residents. These barriers were organized at three levels: resident/ family, health care provider, and system (see Table 2). The document review added further information about organizational policies and procedures related to pain management. When asked about barriers specific to implementing the pain protocol, participants highlighted additional barriers related to its feasibility and ease of implementation.

Residents/Family

Participants identified barriers to pain management at the resident/family level, with a particular emphasis 
Table 2: Qualitative findings about barriers to pain management in LTC

\section{Residents/Family:}

- challenge to assess pain due to:

○ resident's inability to communicate

$\bigcirc$ presence of cognitive impairment

$\bigcirc$ not being able to discriminate pain from other conditions

- $\quad$ reluctance to report pain; residents not wanting to tell staff or family about their pain

- lack of individualized pain treatments based on resident's preferences and side effects of medications

- family's concerns about resident's quality of life, confusion, falls, and dependency on opioids

\section{Health Care Providers:}

- staff not believing resident's verbal report of pain or feel that the resident is overstating his/her pain

- concerns regarding side effects of medications

- reluctance for MD to prescribe pain medications

- the importance of the UCP role regarding resident care, including being a resident advocate

- UCP requires more knowledge and skills to assess pain

- lack of communication between the doctors, NP, and other staff; poor communication within nursing from one shift to another and between UCPs, RN, and NP

- lack of continuity of care; pain medication is ordered and given but then no follow-up

- need for education; lack of specialized skills re: pain management

- problems with prn orders; e.g., med not given when it should be, physician reluctance to order scheduled dosing of pain meds, give too many prn doses when scheduled is needed

- need to use more non-pharmacological interventions

\section{System:}

- time constraints: challenges staff have in providing care to residents due to not enough time

- lack of strong system for pain management to meet accreditation and compliance standards

- appropriate policies and procedures not in place related to pain management

- lack of staff involvement in decision-making about changes in practice but staff state that they want to be involved

- lack of support with changes in practice, e.g., extra staffing, education, hands-on experience

- lack of orientation sessions for new staff about pain management

- lack of resources to implement protocol (e.g., heating pads, music, cold packs)

on the challenges of assessing pain in residents who have cognitive impairment or communication limitations. These residents' lack of ability to report pain created many obstacles for health care providers. Participants shared that it was often difficult to discriminate pain from other common conditions, such as delirium or depression. Consequently, they stated that pain for these residents was not typically managed well.

I think the everyday pain just like you and I take a Tylenol for ... it's always amazing that often that [resident taking a Tylenol] does make a quantitative difference in the restlessness or calling out behaviour ... and it's a fairly innocuous treatment. So I think that kind of everyday [pain] for dementia residents ... when they just sit there ... is that the reason the resident just sits there? because it hurts? ... I don't think we do a good job of managing pain for these kinds of situations.

- Director of Care
For residents who were able verbally to report their pain, other challenges were apparent. Residents described their reluctance to report their pain to LTC staff, stating that they did not want to tell the staff or their family about their pain. For some, pain signified a loss of independence or impending death. As one physiotherapist commented, "Her asking for help was like taking away her independence." Residents did not want to bother LTC staff and would only disclose information about their pain if approached by the nurse first. One resident openly discussed this issue:

Resident: I can be in horrible pain and I don't tell, lots of times I don't tell people because I try to fight it ... but they'll say "oh, you're looking terrible."

Interviewer: Would you tell them how bad it was then?

Resident: If they asked me ... if it was a nurse or something like that, I'd say. 
Interviewer: But you wouldn't tell them [if they didn't ask you], is that what you're saying?

Resident: No, I wouldn't tell them, I wouldn't go tell them, I wouldn't tell them any of this stuff I'm telling you [laughter] ... I've got to live here ...

Interviewer: So you're saying the staff don't always know how much pain you're in and you don't always express that to them.

Resident: Not always, depends on how bad it is. If it's really bad, like the pain is getting really bad, I quite often don't say anything, hoping it will go away.

In addition, residents' level of pain tolerance influenced how their pain was treated; those residents with high levels of pain tolerance would not seek out pain treatments unless absolutely needed. For example, one resident stated:

I'm not a person that takes a lot of medication, if you know what I mean ... I can stand quite a bit of pain like where some people would say, "I have a headache, I need some medication" ... I don't really have that. If I do, I don't really feel it.

Participants commented on the contribution of family members when making decisions related to pain management. Often, family members would be used as a proxy for assessing pain in residents who were unable to provide their own pain reports. In relying on family members, health care providers gained insight into a resident's typical behavioural response to pain, and found this information was most helpful at the time of admission when providers were becoming familiar with the resident. At times, family input, however, presented dilemmas for staff, including some family members' concerns about increased confusion, falls, and opioid dependency associated with resident use of pain medication. A member from the administration stated:

Then we have families who don't want that, saying "morphine kills people", and blah, blah, blah ... we've had residents in pain simply because the family were not willing to go with it ... and I've seen girls [nurses] in tears saying "This isn't right, they [residents] are just so uncomfortable and I don't understand them [family]" ... so it's that kind of thing that I see as the bigger challenge.

Concerns about side effects of pain medications were also expressed by health care providers. For care providers, the importance of individualizing pain interventions was paramount so that the desired balance of pain relief and the side effects of pain medications could be achieved.

RN 1: It's the balance of side effects [that we try to achieve] because I know that we have a few resi- dents where we know that they have pain, but certain pain medications seem to increase confusion, so we are trying to [optimize] quality of life. We don't want them to be in pain but we want them to be lucid so they can meet with their families, and I think that's always an issue in palliative care as well as trying to manage the pain medication effects.

RN2: Or protecting them from falls.

Health care providers

Many staff members commented that they did not always believe residents' verbal reports of pain or they felt that residents were overstating their pain. At times, staff felt they needed to "second guess" the residents' reports of pain.

There's a lot of difficulty in deciding what's official or what's real pain, what's really big pain as you said ... some people say "This is the worst pain I've had in my whole life" without any real sort of physical signs of pain so it's really tough; we have a complex job in assessing that.

Most participants commented on the widespread need for education across health care provider groups and their lack of specialized skills in pain management. They identified a need for knowledge related to using non-pharmacological interventions for pain relief and felt that they should be using these modalities more in their practice. Most participant groups identified a lack of knowledge and skills regarding pain assessment in the UCP group, in particular.

The importance of the UCP role in pain management, specifically with regards to detecting pain in residents, was repeatedly discussed among participants. They stated that often the UCPs would assume an advocacy role for residents.

The [UCP] is the best person to talk to about the cognitively impaired resident's pain because they interact closely with the resident and notice changes in them. - Physiotherapist

RN4: They're [UCPs] very good advocates for the residents, like our [UCPs] are on the residents' side and they will come to you if they have any concerns.

RN2: And they are very caring and they know the residents, they're around all the time.

RN3: We have a very consistent team, and the $[U C P] s$ don't change house, they stay with that group of residents for a long time, they basically know them. They are their eyes and their mouth ... more like family.

Despite this clear acknowledgement of the importance of the UCP role in pain management across all levels of 
health care providers, sometimes the UCPs did not feel supported in their role. UCPs felt that they did not have input regarding decisions involving changes to patient care and, as a result, did not feel empowered in their role in pain management. They were encouraged by their supervisors to report pain, but they were not allowed as unregulated care providers to document it. Unfortunately, sometimes they believed their attempts to report residents' pains were dismissed by the registered nursing staff.

\begin{abstract}
RN: I find one of the biggest challenges here is working with support staff [UCPs] that become very, very anxious ... they assume everything they see is because they [the residents] are in pain they must be in severe pain. And they [the UCPs] come back and report to us "This person is in pain, pain, pain, pain" ... you know, they are actually making a diagnosis, instead of coming back and saying "You know, this person is restless".
\end{abstract}

For the licensed nurses, including RPNs, RNs, and advanced practice nurses, lack of continuity and communication, particularly from one shift to another, regarding pain management was troublesome for them.

RPN: I think poor communication from one shift to another is the biggest problem ... say the resident has had a headache or increased arthritic pain ... just to let that other shift know so they can carry on with giving the analgesic (because arthritic pain on a damp day - they're going to have it all the time) ... so they need to know and then make sure that they are giving the prn medication if it's needed so that they [the residents] are not in a lot of pain.

Poor communication and collaboration related to pain management was also evident between nurses and physicians. Generally, the nursing staff felt that physicians provided adequate orders for residents' pain medication, but sometimes, even though a resident was in constant pain and the staff had documented the pain, the physician prescribed a pro re nata (prn; given as needed) analgesic rather than a regularly scheduled analgesic. This reluctance to order a scheduled medication was amplified when the nurses were working with "non-regular" or on-call physicians. One RPN commented:

A lot of times with the doctor ... if the resident is in pain they [physicians] will order something prn and we're expected to assess them, right ... but if the resident is in pain all the time and receiving this pain medication and it's all documented, they [physicians] still don't want to order it on a regular basis ... so I do have difficulty with residents in constant pain and in need of medication and keeping it at a prn when it should be ordered on a regular basis ... because if someone comes in and doesn't feel like the resident is in pain, it will not be given when in fact he [resident] needs it.

Participants spoke of additional barriers to managing resident pain that were due, in part, to the current staffing model in LTC. Pharmacists stated that not having direct contact with the residents made managing resident pain even more difficult. As a result, they rely on the nurses' clinical skills and observations to inform them if a resident required pain medication.

This model of care where some health care providers are "off-site" had a tendency to impose additional barriers related to poor communication and collaboration among health care providers. Pharmacists expressed concerns related to the follow-up of residents' pain and stated that often they were left wondering if the medication helped alleviate pain because there was no formal communication about treatment effects. Moreover, nurses struggled when they felt that the "current medication wasn't working", stating that residents' pain was not always managed because it took a long time for the physician to prescribe alternate medication.

System

The most common barrier described at the system level was related to time constraints (i.e., challenges for staff to provide resident care due to insufficient time to meet workload demands). For licensed nurses (i.e., RNs, RPNs), documentation requirements were especially troublesome and were perceived to be in place to meet regulatory requirements for compliance and/or accreditation reviews. Staff felt that they needed a strong system for pain management to meet accreditation and compliance standards but were challenged to do so.

RN1: Well, there's a lot of paperwork now [RN2: -oh, yes]; a lot more than there ever was. You have to cover so much [RN2: -yes] ... there's so many assessments you have to do now, and it's weekly. Even the audits in your dining room [RN3: -yeah, dining room], it's audit, audit, audit [RN3: -audit, audit], and on top of that you've got all your new care plans and admissions. On our floor, I'd say we've had about eight or nine admissions in the last three weeks, so you're expected to have a certain part of your care plans done within so many hours and there's just so much time [allotted] to get all that stuff done.

RN2: The pressure from the Ministry [of Health and Long Term Care] down to us and ... some people at the top have no idea how hard we work [RN3: -yeah] how much work there is to be done. We never leave on time, we're always -

RN3: - no taking breaks or lunches.

RN 1: We never take breaks because there's no time; we sneak a sandwich here or there. 
Participants described their lack of involvement in decision making about changes in practice related to pain management as another barrier, but they stated that they would like to be involved in these decisions. Participants elaborated that they were usually made aware of changes in delivery of care via memos and meetings but that they were not always sure why these changes were being made and when the changes would occur, which seemed to be the case for the pain management program. They also said they did not feel that they received enough support, such as extra staffing, education, additional time to learn new skills, or hands-on experience in order to implement the new changes in practice. Health care providers stated that they needed more resources on-site, such as heating pads, music, and cold packs in order to provide good pain management.

Participants highlighted the lack of attention given to pain management during orientation sessions for newly hired staff; they felt that more work needed to be done to equip new staff for dealing with the challenges of managing pain in LTC. They discussed how parts of the pain protocol could be used as a teaching tool during such sessions.

\section{Document Review}

Through the document review, it was evident that both LTC sites had some policies and procedures in place related to pain management although one site had its pain management policies and procedures as a subcomponent of those within palliative care. Upon further inspection of these documents, however, it became evident that there were some gaps consistent between the two LTC homes. Few of the assessment tools included in the policy manuals were evidence based. In fact, one of the included tools had been critiqued extensively in the literature for its lack of validity for use in the older adult population. Moreover, it was not clear which category of health care provider should be responsible for completing specific tasks outlined in the policies and procedures, nor were there directions on how to choose the most appropriate pain scale for a given resident. Still, both of the LTC homes had a behavioural checklist to assess pain in non-verbal residents, consistent with AMDA recommendations.

\section{Specific Feedback about the Pain Protocol}

The majority of participants perceived the pain protocol to be compatible with their current practice and relatively easy to use. An LTC physician recommended a change to one of the original nurse-physician communication strategies (nurse worksheet sent to physicians via fax), stating that faxes are not an effective way to communicate with physicians about residents' pain, but, rather, nurses should use the "Physician Binder" for reporting residents' pain status. Participants felt that the pain protocol would be a good resource for nurses as it would assist in accurate initial assessments, provide more impetus to use non-pharmacological pain interventions, and channel the treatment plan to the most appropriate disciplines. A pharmacist commented:

I think it's good just from what I can see here ... I
think that the information is nicely written and I
think it's easy to interpret and not too cumbersome,
which can be a barrier to pain management, be-
cause if the documentation is too cumbersome
people will try to bypass it all together. So, just by
using the "keep it simple" method, I think that's our
best bet.

Some participants stated that the forms associated with the protocol were too detailed and that the pain protocol added too much paperwork. However, participants stated that they would be more receptive to the pain protocol if they could integrate some of the forms into their current practice. In addition, they stated that they needed to be well educated about using the protocol and would need a lot of support from the "top down" in order to implement it.

Perceptions differed about the general use of protocols in practice. Some participants viewed them as confining because they could deter staff from becoming conceptual thinkers, but at the same time, protocols could be valuable if they could inform and build practice. Others viewed protocols as foundational to improving pain management in their facilities and perceived protocols as a mechanism to promote consistency, continuity, and coordination across health care providers so that resident pain treatment did not "fall through the cracks".

RN: I like protocols ... because, you know, it seems everybody follows the same rules and then if I have to pick up in-between I know where to follow up, and if it is not working we can come back to the protocol and see what is missing.

Administrator: I hate protocols ... I don't like that word ... I think it drives a type of practice I'm not comfortable with ... because I want people [nurses] to be unconsciously competent ... I think that's what I'm saying to you; I want them to just know it. I love them [protocols] if they inform practice and they build practice ... there's a process to get to that and move through all those steps of competence, but I don't want the conscious competence to be the outcome, which is this [pain protocol] it's unconscious.

\section{Discussion and Model Development}

These case study findings highlight many barriers that influence how pain is managed in LTC. The most 
common and frequently reported barriers to pain management were: (a) residents' inability to report pain, (b) residents' reluctance to report pain, and (c) inadequate time for staff to assess pain. These barriers, as well as others, were corroborated by both the quantitative and qualitative findings. The identified barriers to pain management in LTC have also been reported in the literature by others (Kaasalainen et al., 2007; Martin et al., 2005).

A major barrier to managing pain was the assessment of pain in residents with cognitive impairment, which has been well documented in the literature since the early 1990s (Ferrell et al., 1990; Hadjistavropoulos, LaChapelle, MacLeod, Snider, \& Craig, 2002; Herr \& Mobily, 1991; Hurley, Volicer, Hanrahan, Houde, \& Volicer, 1992; Marzinski, 1991; Middleton, Knezacek, Robinson, Hartley, \& Kaasalainen, 1997; Parke, 1992; Parmelee, 1996). In 1991, Marzinski stated that the tragedy of dementia included the possibility that nonverbal older adults were unable to communicate their pain, which may lead to unnecessary suffering. Fortunately since then, growing attention has been focused on developing innovative ways, including many promising tools, to assess pain in this vulnerable population. To date, three systematic reviews have been published that examined such tools (Aubin, Giguere, Hadjistavropoulos, \& Verreault, 2007; Herr, Bjoro, \& Decker, 2006; Zwakhalen, Hamers, Abu-Saad, \& Berger, 2006), all of which concluded that, although further psychometric testing is needed, there are promising tools for use in clinical practice, including the Pain Assessment Checklist for Seniors With Limited Ability to Communicate (PACSLAC) (Fuchs-Lacelle \& Hadjistavropoulos, 2004), Pain Assessment in Advanced Dementia (PAINAD) (Warden, Hurley, \& Volicer, 2003), and the DOLOPLUS-2 (Wary \& Doloplus, 1999).

As dementia progresses, individuals may have increasing difficulty in expressing their pain. For example, Herr et al. (2006) stated that pain can take on "less obvious forms, such as confusion, social withdrawal, aggression, or subtle changes in behaviour, which are not typical pain manifestations" (p. 171). Accordingly, the task of assessing pain becomes more complicated, and the ability of tools to help practitioners discriminate pain from other conditions becomes more difficult. Clearly, there is more work to be done so that pain can be assessed accurately, using standardized tools, in those with varying levels of cognitive impairment.

Another barrier at the resident level that emerged from both the qualitative and quantitative analysis in this study was associated with residents' reluctance to report pain. This reluctance to report pain may be reflec- tive of older adults not wanting to be labeled as "complainers" in LTC which, from their perspective, may affect the care that they would receive from health care providers (Ferrell et al., 1990; Ross, Carswell, Hing, Hollingworth, \& Dalziel, 2001). Alternatively, LTC residents may feel that pain is "a part of the normal aging process" and that by hiding their pain, they could achieve a sense of self-preservation (Blomqvist \& Hallberg, 2001). These findings highlight the need for further patient education, and the need for nurses to encourage residents to discuss their pain experiences in a therapeutic manner. In an attempt to encourage disclosure of resident pain, Arata and Sodickson (2001) posted "pain cards" in each resident's room that included different pain scales, and residents were routinely encouraged to use the cards. In this manner, pain assessment became a normal and accepted part of the routine in LTC, and residents felt more comfortable talking about it with their health care providers. Indeed, pain management needs to become a priority within an organization so that barriers to reporting pain can be minimized.

The importance of the UCP role in managing resident pain was described in this study as being a critical element to detecting resident pain but that these unregulated health care providers lacked the necessary training and knowledge to do so. Mentes, Teer, and Cadogan (2004) reported that, even when UCPs are aware that a resident is in pain, they may not report pain that occurs with caregiving activities, such as during morning care routines (e.g., bathing, dressing, transferring). UCPs need to be encouraged to report pain episodes to supervising nurses so that pain can be minimized by preemptive administration of pain medication or non-pharmacologic interventions (Mentes et al., 2004).

Consistent with our study findings, even when UCPS do report resident pain to their supervisors, their efforts may be ignored, leaving them feeling powerless and demeaned (Mentes et al., 2004). Similarly, Bowers, Esmond, and Jacobson (2003) found that UCPs stated that they felt dismissed or regarded as insignificant by their supervisors and that their skill level was not being recognized. Moreover, UCPs were often excluded from continuing education initiatives within the facility or were not supported by their employers to attend outside educational initiatives (Stolee, Esbaugh, Aylward, Cathers, Harvey, Hillier et al., 2005).

UCPs are in a pivotal position within LTC to recognize pain in residents who have cognitive impairment since UCPs are most responsible for providing care during activities of daily living, which is when pain is most likely to occur (Ferrell et al., 1990). Perhaps, by building capacity within the UCPS by giving them added 
training and tools for practice, they may be more apt to report resident pain in a standardized and consistent manner and to have their reports of resident pain acted upon by their supervisors.

Another prominent barrier from both the qualitative and quantitative findings - lack of time for pain management - was identified as a challenge at the system level, which is consistent with previous research (Bowers, Lauring, \& Jacobson, 2001; Clark, Fink, Pennington, \& Jones, 2006; Kaasalainen et al., 2007; Martin et al., 2005; Resnick, Quinn, \& Baxter, 2004). Bowers et al. (2001) found that nurses' workload, attitude towards work, and patient outcomes were impacted by "time" and suggested that time constraints limit nurses in their ability to provide adequate information and implement evidence-based pain management. Resnick found that workload demands related to documentation were a common challenge when implementing CPGs within 23 LTC facilities (Resnick et al., 2004). In light of these demands, they recommended "keeping it short and sweet" using a step approach to prevent overburdening the implementation sites.

Given that barriers to pain management exist at different levels within the system, it seems logical to use a multi-level approach to address these barriers. A few pain management programs that have targeted multiple levels within the LTC system have been reported with similar barriers identified (Baier, Giofford, Patry, Banks, Rochon, DeSilva et al., 2004; Jones et al., 2004; Resnick et al., 2004; Weissman, Griffie, Muchka, \& Matson, 2001). Generally, these programs propose that pain management must become an organizational priority before any sustainable changes in care processes can be realized. Hadjistavropoulos et al. (2009) outlined key elements within a transformational model of pain management in LTC that include integrating pain assessment and management into a feasible and working clinical model of care, ideally led by a nurse practitioner. Furthermore, LTC facilities should report the results of this clinical model of care to responsible governmental authorities and to the public (Hadjistavropoulos et al., 2009). Spross (2001) argued that more attention needs to be focused on addressing the cultural aspects of organizations in future attempts related to improving pain management in LTC. Although the cultural aspects or barriers are not always explicitly identified, institutional change is a process that requires support and reinforcement (Spross, 2001).

\section{Development of Interdisciplinary Model to Improve Pain Management}

Once the quantitative and qualitative data were analyzed, we developed an approach to modify the pain protocol intervention in light of identified barriers to pain management and the implementation of the pain protocol. Initial drafts of the model were reviewed and refined by the team. Once consensus was reached on the final version of the model, an external pain expert reviewed it for content validity. In addition, we presented it to clinical and administrative representatives from each intervention site.

Based on the quantitative and qualitative findings and drawing from other reported pain management programs (Jones et al., 2004; Weissman et al., 2001; Baier et al., 2004), a multi-tier model was developed with proposed interventions to address identified barriers at each level (see Figure 1). The interventions are organized to highlight accountabilities associated with each level: the resident, family, UCP, licensed health care providers (both on-site and off-site), organization, and system.

Within this model, the resident is represented as the focal point with interacting family and health care provider layers according to the amount of direct contact that each has with the resident while managing resident pain. As depicted in the model, the resident should be encouraged consistently to report pain to health care providers so that an individualized approach to pain management can be developed. If the resident is not able to communicate pain to health care providers, then the family should act as a proxy and receive support from the health care team when needed.

Particular emphasis has been given to the UCP role in pain management within this model. While all groups of participants emphasized the importance of the UCP role to manage resident pain effectively, this role seemed undervalued by some participants. Given the challenges of assessing pain that were described previously, UCPs are in a pivotal position to first recognize it which is a critical step to ensure that pain is addressed. Thus, UCPs need to have the required knowledge, skills, and tools for assessing pain, and they need to assume an empowered role in assessing and reporting pain to licensed nurses. Licensed nurses must be receptive to UCP reports of resident pain, and have the knowledge and skills to use appropriate pain assessment tools and implement both non-pharmacological and pharmacological interventions to manage pain effectively. Resident pain should be evaluated and documented on an ongoing basis and interdisciplinary collaboration optimized using such strategies as care conferencing and interdisciplinary communication tools.

At the organizational level, pain assessment and management needs to be included in the orientation of new staff. Staff should be involved in organizational decisions that affect their pain management practices. 


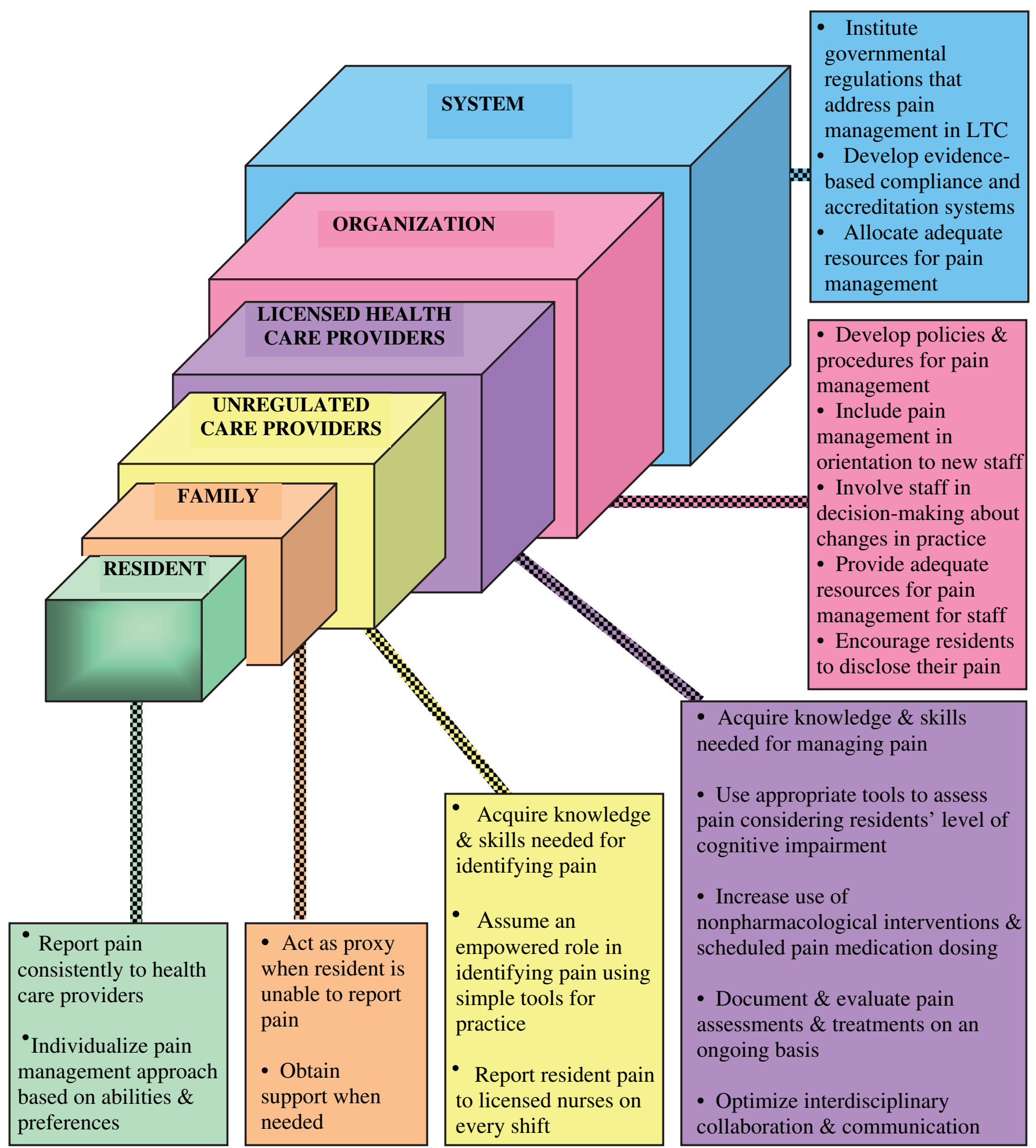

Figure 1: An action-based approach to improving pain management in long-term care (LTC).

Evidence-based policies and procedures should be developed and implemented while allocating adequate resources to do so. A focus at the system level is needed to ensure that pain management is explicitly addressed in governmental regulations and that realistic and evidenced-based compliance and accreditation standards are developed on the basis of these regulations. 
The interdisciplinary model we have proposed here offers a number of recommended actions that may provide some direction for future initiatives aimed at improving pain management in LTC. Strengths of this model lie in its culmination of research findings, from this study as well as other important work, with a particular emphasis on the need for interventions to be directed at specific and multiple levels within the LTC system. This model draws attention to the importance of the UCP role and the need to empower this role within the interdisciplinary team to improve pain management. It highlights the importance of addressing organizational issues and their potential impact on other levels within the model. Future evaluation studies of this model are needed to further assess its validity, applicability, and feasibility in LTC.

To ensure that our findings are generalizable, further research should investigate other LTC facilities in North America and elsewhere, as well as other important health care providers such as social workers, occupational therapists, and speech therapists, to name a few. We did not sample family members directly but, rather, encouraged participants to share their thoughts about perceived barriers to pain management at the family level. Future work should sample this unique group so that their insights and personal experiences can be explored for comparison with these findings.

\section{Conclusions}

Pain management is an important issue in LTC. However, many barriers exist that preclude optimal pain management in this unique setting. Efforts to address these barriers need to focus on multiple levels within the health care system in order for pain management programs to be fully realized. Future research is needed that evaluates ways of treating pain using both pharmacological and non-pharmacological interventions. These interventions need to be housed within an effective knowledge transfer approach that promotes their sustainability in practice. Moreover, the translation of newly developed policy to practice related to pain management needs to be evaluated. In Ontario, for example, new LTC regulations have recently been developed with a particular focus on improving pain management. Future research is needed to examine these changes in policy in terms of their impact on resident outcomes. With more attention given to improving pain management in LTC, it is hoped that unnecessary suffering for this vulnerable population will be alleviated.

\section{References}

American Geriatrics Society. (1998). The management of chronic pain in older persons: AGS panel on chronic pain in older persons. Journal of the American Geriatric Society, 46, 635-651.

American Geriatrics Society. (2009). AGS clinical practice guideline: Pharmacological management of persistent pain in older persons. Retrieved September 13, 2009 from http:/ / www.americangeriatrics.org/education/pharm management.shtml

American Medical Directors Association. (2003). Pain management in the long-term care setting: Clinical practice guideline. American Medical Directors Association.

American Medical Directors Association. (2004). Tool kit for implementation of the clinical practice guideline for pain management.

Arata, M., \& Sodickson, S. (2001). Changing pain management practice at Franciscan Woods. Journal of Palliative Medicine, 4, 575-580.

Aubin, M., Giguere, A., Hadjistavropoulos, T., \& Verreault, R. (2007). The systematic evaluation of instruments designed to assess pain in persons with limited ability to communicate. Pain Research \& Management, 12, 195-203.

Baier, R., Gifford, D., Patry, F., Banks, S., Rochon, T., DeSilva, D., et al. (2004). Ameliorating pain in nursing homes: A collaborative quality-improvement project. Journal of the American Geriatrics Society, 52, 1988-1995.

Bakerjian, D. (2008). Care of nursing home residents by advanced practice nurses. Research in Gerontological Nursing, 1(3), 177-185.

Blomqvist, K., \& Hallberg, I. (2001). Recognizing pain in older adults living in sheltered accommodations: The views of nurses and older adults. International Journal of Nursing Studies, 38, 305-318.

Bowers, B.J., Esmond, S., \& Jacobson, N. (2003). Turnover reimterpreted: CNAs talk about why they leave. Journal of Gerontological Nursing, 29, 36-43.

Bowers, B.J., Lauring, C., \& Jacobson, N. (2001). How nurses manage time and work in long term care. Journal of Advanced Nursing, 33(4), 484-491.

Brown, J.B. (1999). The use of focus groups in clinical research. In B. Crabtree \& W. Miller (Eds.), Doing qualitative research (2nd ed.) (pp. 109-126). London: Sage Publications.

Clark, L., Fink, R., Pennington, K., \& Jones, K. (2006). Nurses' reflections on pain management in a nursing home setting. Pain Management Nursing, 7(2), 71-77.

Crabtree, B., \& Miller, W. (1999). Doing qualitative research (2nd ed). London: Sage Publications.

Desbiens, N., Mueller-Rizner, N., Connors, A., Hamel, M., \& Wenger, N. (1997). Pain in the oldest-old during hospitalization and up to one year later. Journal of the American Geriatrics Society, 45, 1167-1172. 
Duggleby, W. (2005). What about focus group interaction data? Qualitative Health Research, 15(6), 832-840.

Ferrell, B., Ferrell, B., \& Osterweil, B. (1990). Pain in the nursing home. Journal of the American Geriatrics Society, $38,409-414$

Fox, P., Raina, P., \& Jadad, A. (1999). Prevalence and treatment of pain in older adults in nursing homes and other long-term care institutions: A systematic review. Canadian Medical Association Journal, 160, 329-333.

Fuchs-Lacelle, S., \& Hadjistavropoulos, T. (2004). Development and preliminary validation of the pain assessment checklist for seniors with limited ability to communicate (PACSLAC). Pain Management Nursing, 5, 37-49.

Gilchrist, V., \& Williams, R. (1999). Key informant interviews. In B. Crabtree \& W. Miller (Eds.), Doing qualitative research (2nd ed.). London: Sage Publications.

Hadjistavropoulos, T., LaChapelle, D., MacLeod, F., Snider, B., \& Craig, K. (2002). Measuring movementexacerbated pain in cognitively impaired frail elders. Clinical Journal of Pain, 16, 54-63.

Hadjistavropoulos, T., Marchildon, G., Fine, P., Herr, K., Palley, H., Kaasalainen, S., et al. (2009). Transforming long-term care pain management in North America: The policy-clinical interface. Pain Medicine, 10(3), 506-520.

Herr, K., Bjoro, K., \& Decker, S. (2006). Tools for assessment of pain in nonverbal older adults with dementia: A state-of-the-science review. Journal of Pain and Symptom Management, 31, 170-192.

Herr, K., \& Mobily, P. (1991). Complexities of pain assessment in the elderly. Journal of Gerontological Nursing, 17(4), 12-19.

Horgas, A., \& Tsai, P. (1998). Analgesic drug prescription and use in cognitively impaired nursing home residents. Nursing Research, 47(4), 235-242.

Hurley, A., Volicer, B., Hanrahan, P., Houde, S., \& Volicer, L. (1992). Assessment of discomfort in advanced Alzheimer patients. Research in Nursing and Health, 15, 369-377.

Jones, K., Fink, R., Pepper, G., Hutt, E., Vojir, C., Scott, J., et al. (2004). Improving nursing home staff knowledge and attitudes about pain. The Gerontologist, 44(4), 469-478.

Kaasalainen, S., Coker, E., Dolovich, L., Papaioannou, A., Hadjistavropoulos, T., Emili, A., et al. (2007). Pain management decision-making among long-term care physicians and nurses. Western Journal of Nursing Research, 29(5), 561-580.

Kaasalainen, S., \& Crook, J. (2003). A comparison of painassessment tools for use with elderly long-term care residents. Canadian Journal of Nursing Research, 35(4), 58-71.

Kaasalainen, S., Middleton, J., Knezacek, S., Stewart, N., Hartley, T., Ife, C., et al. (1998). Pain and cognitive status of institutionalized elderly: Perceptions and interventions. Journal of Gerontological Nursing, 24(8), 24-31.
Martin, R., Williams, J., Hadjistavropoulos, T., Hadjistavropoulos, H., \& MacClean, M. (2005). A qualitative investigation on seniors' and caregivers' views on pain assessment and management. Canadian Journal of Nursing Research, 37(2), 142-164.

Marzinski, L. (1991). The tragedy of dementia: Clinically assessing pain in the confused, nonverbal elderly. Journal of Gerontological Nursing, 17, 25-28.

Medical College of Wisconsin. (2000). Nursing staff education resource manual: Pain management 101 in long-term care facilities. Milwaukee, WI: The Medical College of Wisconsin.

Mentes, J.C., Teer, J., \& Cadogan, M. (2004). The pain experience of cognitively impaired nursing home residents: Perceptions of family members and certified nursing assistants. Pain Management Nursing, 5(3), $118-125$

Middleton, J., Knezacek, S., Robinson, L., Hartley, T., \& Kaasalainen, S. (1997). An exploratory study of pain in the institutionalized elderly. American Journal of Alzheimer's Disease, 12(4), 159-166.

Morgan, D. (1997). Focus groups as qualitative research (2nd ed.). London: Sage Publications.

Moulin, D., Clark, A., Speechley, M., \& Morley-Forster, P. (2002). Chronic pain in Canada-Prevalence, treatment, impact and the role of opioid analgesia. Pain Research $\mathcal{E}$ Management, 7(4), 179-184.

Parke, B. (1992). Pain in the cognitively impaired elderly. Canadian Nurse, 88(7), 17-20.

Parmelee, P. (1996). Pain in cognitively impaired older persons. Clinics in Geriatric Medicine, 12(3), 473-487.

Patton, M.Q. (2000). Qualitative research and evaluation methods (3rd ed). Thousand Oaks, CA: Sage.

Proctor, W., \& Hirdes, J. (2001). Pain and cognitive status among nursing home residents in Canada. Pain Research $\mathcal{E}$ Management, 6, 119-125.

QSR International Ltd. (2007). N Vivo (Version 7.0). Thousand Oaks, CA: Scolari-Sage Publications Software.

Registered Nurses Association of Ontario. (2002a). Nursing best practice guideline: Assessment and management of pain. Registered Nurses Association of Ontario, Toronto, ON. Retrieved April 11, 2009, from http://www.rnao.org/ Storage/11/543BPG_assessment_of_pain.pdf

Registered Nurses Association of Ontario. (2002b). Toolkit: Implementation of clinical practice guidelines. Toronto, Ontario, Canada: Author. Retrieved March 2002, from http:/ / www.rnao.org / bestpractices / completed_ guidelines/BPG_Guide_C1_Toolkit.asp

Resnick, B., Quinn, C., \& Baxter, S. (2004). Testing the feasibility of implementation of clinical practice guidelines in LTC facilities. Journal of the American Medical Directors Association, 5(1), 1-8. 
Ross, M., Carswell, A., Hing, M., Hollingworth, G., \& Dalziel, W. (2001). Seniors' decision making about pain management. Journal of Advanced Nursing, 35(5), 442-451.

Ross, M., \& Crook, J. (1998). Elderly recipients of home nursing services: Pain, disability and functional competence. Journal of Advanced Nursing, 27, 1117-1126.

Sandelowski, M. (2000). Whatever happened to qualitative description? Research in Nursing \& Health, 23, 334-340.

Simons, W., \& Malabar, R. (1995). Assessing pain in elderly patients who cannot respond verbally. Journal of Advanced Nursing, 22, 663-669.

Spross, J. (2001). Harnessing power and passion: Lessons from pain management leaders and literature. Journal of Pain Medicine, 4(4), 557-566.

Stevens, P. (1996). Focus groups: Collecting aggregate-level data to understand community health phenomena. Public Health Nursing, 13, 170-176.

Stolee, P., Esbaugh, J., Aylward, S., Cathers, T., Harvey, D., Hillier, L., et al. (2005). Factors associated with the effectiveness of continuing education in long-term care. The Gerontologist, 45(3), 399-405.

Warden, V., Hurley, A.C., \& Volicer, L. (2003). Development and psychometric evaluation of the Pain Assessment in Advanced Dementia (PAINAD) scale. Journal of the American Medical Directors Association, 4, 9-15.

Wary, B., \& Doloplus, C. (1999). Doloplus-2: Une échelle pour évaluer la douleur [Doloplus-2: A scale for pain measurement]. SoinsGérontologie, 19(August-October), $25-27$.

Weissman, D., Griffie, J., Muchka, S., \& Matson, S. (2001). Improving pain management in long-term care facilities. Journal of Palliative Medicine, 4(4), 567-573.

Yin, R. (1984). Case study research: Design and methods. London: Sage Publications.

Zwakhalen, S., Hamers, J., Abu-Saad, H., \& Berger, M. (2006). Pain in elderly people with severe dementia: A systematic review of behavioral pain assessment tools. BMC Geriatrics, 6(3), 1-15. 
AUTHOR QUERIES

\begin{tabular}{|l|l|}
\hline 1 & Please provide mots clés for this article. \\
\hline
\end{tabular} 